\title{
How does speed change affect induced motion?
}

\author{
ROBERT BECKLEN \\ Sarah Lawrence College, Bronxville, New York \\ and \\ HANS WALLACH \\ Swarthmore College, Swarthmore, Pennsylvania
}

\begin{abstract}
The nature of a recently reported effect of speed on induced motion was investigated. Wallach and Becklen (1983) had found that the extent of induced motion decreases linearly with increases in presentation speed. We found that this speed effect occurred only in induced motion and not under conditions in which configurational change, which is responsible for induced motion, operated veridically. It took place whether configurational change was in conflict with ocular pursuit or with image displacement, and seemed to consist in diminished effectiveness of configurational change when it competed with one of these subject-relative stimuli.
\end{abstract}

We have previously reported (Wallach \& Becklen, 1983) that induced motion became diminished when the motion of the pattern that produced it was made faster. In our experiments, a pattern that moved horizontally back and forth caused induced motion of a dot in its center. When the reciprocating motion of the pattern was made faster, the extent of the dot's apparent motion became smaller. As was done in earlier experiments in which the extent of induced motion was measured, we did not obtain direct estimates of the extent of the induced motion but, instead, measured the shape of the motion path that resulted when we allowed the induced motion to combine with a real motion of the dot. Wallach, Bacon, and Schulman (1978) made the dot in the center of the moving pattern that caused its induced motion move up and down when the inducing pattern moved left and right. The pattern consisted of evenly spaced vertical lines long enough for their ends not to serve as landmarks for the vertical motion of the dot. Therefore, the real motion of the dot was given only as ocular pursuit when the subjects were permitted to track its motion. Since both the line pattern and the dot reversed their respective motions simultaneously, the dot's horizontal induced motion always started and reversed direction together with its real vertical motion. Therefore, the two motions combined to form an oblique path whose slope depended on the extent of the induced motion and the extent of the real motion of the dot. When the extent of the real motion was kept constant, the slope angle of the dot's apparent motion path was a measure of the extent of the induced mo-

This work was supported by Grant 11089 from the National Institute of Mental Health to Swarthmore College, Hans Wallach, principal investigator. R. Becklen's mailing address is: Department of Psychology, Sarah Lawrence College, Bronxville, NY 10708. H. Wallach's is: Department of Psychology, Swarthmore College, Swarthmore, PA 19081. tion. The perceived sloping path was the psychological analogue to the simplest Lissajous path that is obtained when an object is engaged simultaneoulsy in two reciprocating motions whose directions are different. When the motions are in phase, the combined motion, the kinematic resultant of the two component motions, is a straight path.

Wallach and Becklen (1983) used a similar method for measuring the extent of induced motion when they demonstrated the effect of speed on induced motion. They changed the phase relationship between the dot and the line-pattern motion so that one motion started when the other was at its midpoint. Physical motions so combined result in an elliptical or circular Lissajous path, and similar motion paths are perceived when a horizontal induced motion and a vertical real motion given by ocular pursuit combine psychologically. Here the shape of the perceived oval path can be used as a measure of the extent of the induced motion: The width of the oval represents the extent of the induced motion, and its height the extent of the vertical dot motion. When speeding up the motions in the display diminished the induced motion, the perceived oval path became narrower.

This speed effect was not caused by the blurring, at high speeds, of the line pattern, whose horizontal motions were given as displacements of its image across the retina when the eyes tracked the dot. Wallach and Becklen (1983) found that replacing the sharp line pattern with a strongly blurred one did not diminish the extent of the induced motion. They also found that no genuine speed effect was obtained when the eye tracked the line pattern instead of the dot. To explain this, the authors conjectured that no induced motion took place when the lines were tracked. In that case, the vertical motion of the dot formed an oval path on the retinas, the resultant of the dot's objective motion and the horizontal eye movements, and this oval retinal path directly caused the perceived oval path, which, 
therefore, was not a manifestation of induced motion. This conjecture was later tested and supported by Becklen, Wallach, and Nitzberg (1984).

The experiments reported here further explored the speed effect.

\section{Does speedup affect all motion that involves configurational change or only induced motion?}

Induced motion is caused by a relative displacement between an object and its background typically when the object is stationary and the background is moving. The same kind of stimulation represents the real motion of an object when that motion is given as the object's displacement relative to a stationary background pattern. In both cases, stimulation consists of the changing configuration in the retinal projections of the scenes. Such configurational change between an object and its visual surround usually leads to a perception of motion. When the object really moves and the environment is stationary, veridically perceived motion of the object always results. When the object is stationary and the surround moves, induced motion often occurs. In the latter case, the outcome is the result of a conflict between two kinds of stimulation, the configurational change that causes the object's tendency toward motion and the stimulation conditions with which the immobility of the object is given. The latter are the so-called subject-relative stimulus conditions that represent the change in location of the moving object relative to the observer; ocular pursuit represents motion when the eyes track the moving object, and displacement of the moving object's retinal image does so when the eyes are fixed on a stationary point. Because these subject-relative stimuli represent the object's immobility, induced motion is not always seen. For unknown reasons, induced motion is always perceived when the object has a motion of its own, and induction causes the direction of that motion to be altered.

Since both induced and veridical motion result from the same-kind of stimulation, namely, configurational change, the following question arises: Does speedup have an effect on all motion that results from configurational change, that is, on veridical motion perception also, or is it restricted to induced motion? Our first two experiments were done to provide an answer to this question.

\section{EXPERIMENT 1}

The effect of varying motion speed on the extent of perceived motion was measured under three experimental conditions: (1) for induced motion, (2) for real motion on a patterned background where it was given as configurational change, and (3) for real motion on a homogeneous background where it was given by one of the subject-relative stimulus conditions. The last condition was needed because, unless the motion is very slow, one of the subject-relative stimulation conditions always operates in addition to configurational change when real motion is given on a patterned background. In the two conditions in which real motion was observed, we had the subject track the moving object so that its subjectrelative motion was given as ocular pursuit. We selected ocular pursuit to represent the subject-relative displacement of the real motion rather than image displacement, because it is a less potent stimulus for motion than image displacement (Wallach, O'Leary, \& McMahon, 1982). Therefore, the perception of the real motion on a patterned background would depend to a greater degree on configurational change, and less on unavoidable subjectrelative stimulation. Wallach et al. (1982) had also found that ocular pursuit is much less effective than configurational change when the two stimuli conflict.

The following proximal stimuli were operating in our three experimental conditions. In the induced motion condition (I), configurational change caused the perceived motion of the dot, but since it was objectively stationary, the subject-relative stimuli represented it as stationary and thus provided conflicting inputs. When the object's motion was real and seen on a patterned background, it was given veridically by configurational change and by ocular pursuit (CP). Finally, when the moving object was observed in a homogeneous field, its motion was given by ocular pursuit only (P). This condition was needed as a control because circular motion that results from ocular pursuit has been shown to be subject to a speedup effect as well (Coren, Bradley, Hoenig, \& Girgus, 1975).

The expectations for our experiments were as follows: If speedup affects induced motion exclusively, any speedup effect that shows up in the CP condition could only occur because of its above-mentioned effect on motion given by pursuit, and its effect in the CP condition could not be stronger than its effect in the $P$ condition. If, on the other hand, speedup also affects other instances of configurational change, its effect in the CP condition should be stronger than in the $\mathrm{P}$ condition.

Because it yields better measurements of motion extent, we used a circular motion path in all three conditions. The apparent diameter of this circle was the dependent measure used.

\section{Method}

Subjects. Fourteen paid undergraduates of Swarthmore College served as subjects.

Equipment. The device used by Wallach and Becklen (1983) to present a horizontally moving line pattern and a vertically moving dot in concert and with a $90^{\circ}$ phase shift was modified. That device moved two projector beams in simple harmonic and reciprocating motion by means of tilting mirrors, one of which moved a beam horizontally while the other moved a second beam vertically. Now stationary mirrors were added that caused the two moving mirrors to shift the same beam successively. Since the two beam motions that were combined in this fashion were of the same extent, the resulting motion of the single beam was circular. It came to a focus on a translucent screen on which it was projected from the rear. In the real-motion conditions, $\mathbf{P}$ and $\mathbf{C P}$, the moving beam projected a bright dot $5 \mathrm{~mm}$ across, which moved on a circular path $9 \mathrm{~cm}$ in diameter. In the induced-motion condition, I, the moving beam projected a square pattern of dots turned into the diamond orientation, with all dots moving on 9-cm circular paths. The dots of the pattern were dark on a light ground and $5 \mathrm{~mm}$ in diameter, 
and the distances between their centers were $2.0 \mathrm{~cm}$ in either direction. The pattern filled an area of $100 \times 100 \mathrm{~cm}$. In this condition, the bright dot was projected by a stationary beam from another lantern into the center of the field. In the CP conditions, the pattern of dark dots was projected by a stationary beam. The observation distance was $40 \mathrm{~cm}$, and the circular path, therefore, subtended an angle of approximately $13^{\circ}$.

Procedure. Each condition was presented at three speeds. One complete circular motion of the bright dot or of the dots in the pattern took $3.85,1.89$, or $1.25 \mathrm{sec}$, corresponding to motion speeds of $.26, .53$, and .8 cycles/sec (cps). In all conditions, the subjects were instructed to keep the bright dot at the center of their vision at all times, which amounted to tracking it in the P and CP conditions and fixating it in the I condition. Estimates of the size of the circular motion paths were made by adjusting a pair of calipers until the distance between the tips conformed to the diameter of the perceived motion path.

The order of presentation of the three conditions was counterbalanced across 12 of the subjects, and the remaining two were given the conditions in the order of two earlier ones. In each condition, the speeds were presented either in ascending or descending order; half the subjects started with ascending order, performed the next condition in descending order, and did the last condition in ascending order. The other subjects started the alternation with the descending order. One size estimate was given after each presentation. When this sequence was completed, the entire design was repeated in reverse order after a short rest period, and a second estimate was obtained for each of the nine combinations of condition and speed. The average of the two estimates obtained for one such combination became a score.

\section{Results}

These scores, which represent the estimates of the diameters of the dot's circular path, were divided by 9 , the objective diameter of the path in two of the conditions. The means of these ratios are listed in Table $1 .^{1}$ In Condition CP, the mean estimate of the diameter at the lowest speed was very close to the diameter of the objective path, and higher speeds affected the apparent size of the circular path only in a minor way. When, in Condition $P$, the motion of the dot was given without the background pattern, the size diminished somewhat more than in the CP condition as speed increased. This size change was a manifestation of the above-mentioned effect of speed on the apparent size of a circular motion path that is given by ocular pursuit. The mean slopes of the functions relating speed and size estimates, represented by the ratio scores, are shown in the last column of Table 1. There was a clear difference among the slopes $[F(2,26)=6.10, p=.007]$. Post hoc $t$ tests showed that Condition I had a significantly steeper drop than either of the other two conditions, which did not differ from one

Table 1

Mean Estimates of the Diameter of the Dot's Circular Path Obtained Under Three Conditions and At Three Speeds, Given as Proportion to Objective Diameter of $9 \mathrm{~cm}$

\begin{tabular}{lccccc}
\hline & \multicolumn{5}{c}{ Speed in cps } \\
\cline { 2 - 6 } \multicolumn{1}{c}{ Conditions } & .26 & .53 & .80 & av. & Slopes \\
\hline Configurational + Pursuit (CP) & .97 & .92 & .89 & .92 & -.15 \\
Pursuit (P) & .89 & .79 & .76 & .81 & -.25 \\
Induced Motion (I) & .77 & .65 & .52 & .65 & -.46 \\
\hline
\end{tabular}

another. The critical comparison, between Conditions CP and $\mathrm{I}$, was highly significant $[\mathrm{t}(26)=3.42, \mathrm{p}=.002]$. These differences in the slopes of the size estimate means make it clear that motion path sizes dropped substantially faster in the I condition than in the CP condition. The speed effect connected with configurational change was quite large in the I condition but small when, in the CP condition, configurational change operated in veridical perception. The small speed effect that did occur in the CP condition is accounted for by the speed effect on pursuit, which was measured in Condition P. In fact, when in the CP condition configurational change operated in concert with ocular pursuit, it counteracted the speed effect that affects ocular pursuit; motion path sizes were consistently and significantly larger in the $\mathrm{CP}$ condition than in the P condition. An ANOVA for the averaged motion path sizes that were obtained under the three conditions (shown in the fourth column of Table 1) showed $\mathrm{F}(2,26)=15.58, \mathrm{p}<.00004$. A planned-comparison $t$ test for the difference between the averaged motion path sizes of Conditions $\mathrm{CP}$ and $\mathrm{P}$ yielded $\mathrm{t}(26)=2.24, \mathrm{p}=$ .04. It appears that the speed effect connected with configurational change occurred only in the case of induced motion.

\section{EXPERIMENT 2}

Our next experiment was designed to demonstrate the correctness of this inference more directly. In a new display, configurational change represented a real vertical motion and, at the same time, caused induced horizontal motion. The display used by Wallach and Becklen (1983) was modified. In that display, a pattern of horizontally moving vertical lines induced a horizontal motion component in a vertically moving dot. Since the vertical lines provided no landmarks for the vertical motion of the dot, that motion evoked no configurational change and was given by ocular pursuit only. As previously stated, the two motions were combined in such a way that their combined path was circular or oval. In the present experiment, the line pattern was replaced by a regular dot pattern similar to the one that was used in our Experiment 1. It provided the same density of landmarks for the real vertical motion as for the relative displacement between the dot and the horizontally moving pattern. The vertical and the horizontal motions were thus given by identical configurational changes. It should not matter that the configurational change in the vertical dimension mediated real motion, that is, that it provided the same information as ocular pursuit, whereas in the horizontal dimension it caused induced motion and was in conflict with ocular pursuit, because the latter represented vertical motion. ${ }^{2}$ If speedup diminishes the effect of configurational change under all circumstances, it would tend to diminish both the vertical and the horizontal component of the dot's path, and both components would undergo a change. If, on the other hand, speedup diminishes only induced motion, only the width of the dot's path should become smaller at higher 
speeds. The possibility that speedup might also affect the vertical motion that resulted from pursuit of the vertically moving dot, analogous to the effect on circular motion we had obtained in Condition P of Experiment 1, was not of concern here, because our earlier work on the effect of speed on induced motion had shown no speed effect on the motion that resulted from pursuit of a dot in straight vertical motion. ${ }^{3}$

\section{Method}

Subjects. The subjects were 12 paid undergraduates.

Equipment. The device used in Experiment 1 was changed back to the way it was used by Wallach and Becklen (1983). The moving mirrors shifted different projector beams. The vertically moving beam projected the bright dot and the horizontally moving beam a regular pattern of black dots similar to the one used in Experiment 1 . The bright dot measured $7 \mathrm{~mm}$ across and moved $9.2 \mathrm{~cm}$ up and down. The black dots in the pattern were $10 \mathrm{~mm}$ in diameter, and the horizontal and vertical distances between their centers were $3 \mathrm{~cm}$. The dot pattern field was $95 \times 95 \mathrm{~cm}$, and the excursion of its horizontal reciprocating motion was $8 \mathrm{~cm}$. The observation distance was $40 \mathrm{~cm}$. As before, one motion always started when the other was at its midpoint so that the combination of the dot's objective vertical motion with its induced horizontal motion resulted in an oval perceived path.

Procedure. The subject gave estimates of the height and of the width of this motion path by setting the gap in a pair of calipers. The display motion was observed at four velocities, the three speeds that were used in Experiment 1, and a higher velocity of $1.07 \mathrm{cps}$. Half the subjects gave their height and width estimates in ascending order of speed, and the other half gave them in descending order.

\section{Results}

The mean height and width estimates that were made at the four speeds are listed in Table 2 . They showed a clear result. The height estimates remained unaltered, but the width estimates declined steadily as speed increased. Each speedup step caused a significantly smaller mean width estimate with the values of $t(33)$ for the three steps amounting to $3.54,2.85$, and 3.78 , respectively [planned comparison ts following significant ANOVA: $F(3,33)=$ $37.4, p<.008]$. The slope of the mean width estimates in relation to speed was -.50 , which is close to the -.46 value obtained in the I condition of Experiment 1 . Speedup diminished only the horizontal dimension of the perceived motion path; its vertical dimension remained unaltered. Configurational change operated in both dimensions, but only the horizontal motion component was given as induced motion. The speedup effect was again shown to occur only in connection with induced motion, and not where configurational change resulted from real motion.

Table 2

Mean Width and Mean Height Estimates of Oval Motion Path at Four Speeds (in Centimeters)

\begin{tabular}{lcccc}
\hline & \multicolumn{5}{c}{ Speeds in cps } \\
\cline { 2 - 5 } & .26 & .53 & .80 & 1.07 \\
\hline Height & 7.47 & 7.40 & 7.42 & 7.37 \\
Width & 7.17 & 5.99 & 5.12 & 3.91 \\
\hline
\end{tabular}

Experiments 1 and 2 both indicated that speed change affects only induced motion.

\section{Does speedup diminish induced motion because it makes ocular pursuit more effective?}

Induced motion is distinguished from other instances in which configurational change is a stimulus for motion in that it involves an informational conflict with other conditions of stimulation. In Experiment 2, ocular pursuit represented the dot's objective vertical motion and was in conflict with configurational change, which caused the dot to move horizontally. The speedup effect in induced motion could be related to either of the two stimulus conditions in conflict: it could result from a configurational change's becoming less effective as speed increases or it might be a case of ocular pursuit becoming more effective. Ocular pursuit may well be more effective when the eye movements that are registered are more rapid. Our last experiment put this possibility to a test.

\section{EXPERIMENT 3}

Ocular pursuit was eliminated as the competing condition of stimulation, and the objective vertical motion of the dot was instead given as image displacement. If the speed effect was caused by ocular pursuit's becoming more effective, it should now fail to take place. The opposite result, that speedup still diminished the horizontal dimension of the motion path, would favor the conclusion that speedup makes configurational change less effective. Only the unlikely assumption that speedup makes image displacement also more effective would make this conclusion invalid.

Experiment 3 employed the display used by Wallach and Becklen (1983) to investigate the speed effect. In this display, a pattern of vertical lines moved horizontally and caused induced motion of a bright dot that moved vertically. The two motions were combined to yield a circular or oval perceived path. In all previous experiments in which this display was used, the vertical motion of the dot was tracked; now subjects fixated a stationary mark, aligned with and near the upper end of the dot's path. As a consequence, the dot's path was now given as an image displacement rather than through pursuit movements. Wallach et al. (1982) had found that when, in induced motion displays, configurational change conflicted with image displacement, the two stimuli were about equally effective; configurational change had about one-half of the effect it had when it was in conflict with ocular pursuit, in which it was fully effective. In the experiments by Wallach et al. (1982), the objective motion of the dot and its induced motion were so combined that they started and stopped together, and the perceived path was linear and oblique. Because that path was oval in our display, we added as controls the two observation conditions that Wallach et al. (1982) had used, but employed conditions 
that made the perceived path oval. In both control conditions, the dot's motion was tracked. In one, the stationary mark that was fixated in the image displacement condition was present, and in the other it was absent. Adding these conditions also made it possible to compare our results with those obtained by Wallach et al. (1982) and by Wallach and Becklen (1983). A further difference between these earlier experiments and the present one was that we now used different motion extents for the dot and the line pattern rather than paths of equal lengths as had been done before. Because we expected the effect of the line pattern motion to be diminished in the image displacement condition, we made its extent 1.24 times larger than the extent of the dot motion.

\section{Method}

Subjects. The subjects were eight paid undergraduates.

Procedure. The bright dot measured $5 \mathrm{~mm}$ across and moved $10.4 \mathrm{~cm}$ up and down. The vertical lines that made up the pattern were $96 \mathrm{~cm}$ tall, $6 \mathrm{~mm}$ wide, and $1.5 \mathrm{~cm}$ apart, and their horizontal excursion was $12.9 \mathrm{~cm}$. The stationary mark measured $4 \mathrm{~mm}$ across and was $2 \mathrm{~cm}$ above the upper end of the dot's path. The observation distance was $50 \mathrm{~cm}$, and the four motion speeds that were used in Experiment 2 were presented in either ascending or descending order. At each speed, a subject gave a width and a height estimate, using a pair of calipers. The experiment always started with the fixation condition, followed by the two pursuit conditions, which were presented in counterbalanced order across subjects. After this, the fixation condition was once again repeated.

Speedup was expected to diminish the horizontal component of the oval motion path and, therefore, to diminish the ratio of the width and height estimates in the two pursuit conditions (with and without the stationary dot present). What would happen in the fixation condition was the issue of this experiment.

\section{Results}

For each pair of estimates, a width/height ratio was formed and became a subject's score for a particular combination of motion speed and observation condition. The means of these scores are listed in Table 3, where the means obtained in the two fixation trials have been combined, since the order effect was negligible. In the two pursuit conditions, the effect of speeding up the motions was even somewhat greater than in the corresponding condition of Wallach and Becklen's (1983) Experiment 1. The average slopes of the drop in the $\mathrm{w} / \mathrm{h}$ ratios in the present experiment were -.64 and -.69 , while a slope of -.46 had been obtained in the previous experiment. The results of the pursuit conditions as well as the result of the fixation condition were also in agreement with the results ob-

Table 3

Mean Width/Height Ratios Obtained in Experiment 3 Under Three Observation Conditions at Four Motion Speeds and at $.17 \mathrm{cps}$ by Wallach, O'Leary, and McMahon

\begin{tabular}{lrrrrrr}
\hline & \multicolumn{6}{c}{ Speed in cps } \\
\cline { 2 - 7 } & .26 & .53 & .80 & 1.07 & Av. & .17 \\
\hline Stationary, mark fixated & .65 & .56 & .43 & .32 & .49 & .44 \\
Pursuit, mark present & .86 & .73 & .51 & .37 & .62 & .66 \\
Pursuit, mark absent & 1.24 & 1.05 & .89 & .67 & .96 & 1.05 \\
\hline
\end{tabular}

tained by Wallach et al. (1982). The results of their Experiment 2 are listed in the last column of our Table 3 under the heading " .17 ," which was the motion speed they used. In our pursuit condition with the dot absent, the mean $\mathrm{w} / \mathrm{h}$ ratio, that is, the ratio of the extents of the horizontal and vertical components, was 1.24 at the lowest speed. This ratio was identical to the ratio of the objective extents of the motions of the inducing pattern and of the dot, indicating that the configurational change that brought about the induced motion was fully effective at this speed. The earlier authors had likewise obtained complete induced motion, with a mean judged ratio of 1.05 of the extents of the horizontal and vertical components, as against an objective ratio of 1.0.

Our results in the two viewing conditions in which the stationary mark was present can be compared with those obtained by Wallach et al. (1982). Because the extents of the line pattern motions were different in the two experiments, we computed for both studies the proportion of the mean ratio for pursuit with the stationary mark present over the mean ratio for pursuit in the absence of the mark, and the proportion of the mean ratio for the fixation condition also over the mean ratio for pursuit in the absence of the mark. This provided an estimate of the effect of the stationary mark as such, under pursuit and fixation. In our experiment, this proportion was .86/1.24, or .69 , for pursuit with mark present, and $.65 / 1.24$, or .52 , both at the slowest speed, for the fixation condition. In the previous experiment, the corresponding proportions had been $.66 / 1.05$, or .63 , and $.44 / 1.05$, or .42 . That these proportions were in fair agreement was important, since the ratios of the extents of the horizontal and vertical components of the perceived motion paths were measured in different ways, because the motion paths were different in the two experiments. In the previous experiment, the paths that resulted from the real vertical motion and the induced horizontal motion were sloping straight lines and the ratios of the components were represented by the tangents of the estimated slope angles, whereas in our experiment, the paths were ovals and the ratios of the components were represented by the ratios of the estimates of their widths and heights.

The main result of our experiment was the decrease of the mean $w / h$ ratios in the fixation condition as motion speed increased. The means listed in the first row of Table 3 showed a slope of the drop of the mean $w / h$ ratios that amounted to -.42 and was significantly different from zero at the .004 level by the binomial test. This speed effect was comparable to previous estimates obtained under target fixation and pursuit conditions, which ranged between -.45 and -.50 (e.g., Experiments 1 and 2 in this report, and Wallach \& Becklen, 1983). Although the extents of induced motion were only half as large in the image displacement condition as in the pursuit condition (third row), the slopes representing the relationship between speed and extent of induced motion were similar. Having the vertical motion of the dot be given as image 
displacement rather than by ocular pursuit did not alter the speed effect. Together with Experiment 1, these results show that the speed effect is not a matter of ocular pursuit. It is, rather, very likely that speedup in some way alters configurational change when the latter causes induced motion.

\section{SUMMARY AND CONCLUSIONS}

Induced motion results from configurational change. That an increase in motion speed diminishes the extent of induced motion may therefore be a manifestation of speedup's altering all perceived motion that involves configurational change. If that were the case, an increase in motion speed should also diminish the extent of perceived real motion. Experiments 1 and 2 demonstrated that this is not so, and that speedup alters only induced motion.

Induced motion is different from perceived real motion that results from configurational change in that, in induced motion, configurational change is in conflict with subjectrelative stimuli, with ocular pursuit in that role in Experiments 1 and 2 . Since speedup was found to have an effect only in induced motion, the question arose as to which partner in the conflict it affects. Is configurational change made less effective or ocular pursuit more? It seemed possible that more rapid eye movements would be advantageous when ocular pursuit served as stimulus for motion perception. In Experiment 3, we omitted ocular pursuit and had the real motion given as image displacement. We found that speedup still diminished induced motion. Although configurational change is only half as effective when it is in conflict with image displacement, speedup diminished induced motion at the same rate as when configurational change was in conflict with ocular pursuit. This made it likely that speedup affected induced motion by affecting configurational change. As previously discussed, the result of Condition I of Experiment 1 supported this conclusion.

The fact that speedup has an effect only on induced motion could be the outcome of two subtly different processes. One possibility is that the effect depends intrinsically on the conflict inherent in induced motion and that, for unknown reasons, configurational change becomes less effective with increased speed only if it conflicts with the information given by ocular pursuit or image displacement. Where configurational change and subject-relative information are in agreement, no conflict exists and hence no basis for the speed effect. Thus, veridical perception normally results.

The second explanation assumes that faster configurational change always tends to lessen perceived motion extents, although under normal conditions the final perceptual outcome is so dominated by subject-relative stimulation that the speed effect is essentially masked. Consequently, it is measurable with present procedures only under induced-motion conditions.
The basic speed effect reported in Wallach and Becklen (1983) is compatible with either explanation. The second alternative might be preferred because the assumption that faster configurational change always lessens perceived motion extents is clearer and simpler. But is is incompatible with the result of Experiment 1, in which real motion (CP) showed significantly less of a speed effect than either ocular pursuit (P) or induced motion (I). If it were true that, in real motion perception, the extent of apparent motion depended more on subject-relative change with increased speed, then the speed effect on ocular pursuit measured in Condition P should have been fully effective in Condition CP as well, but it was not. It is thus necessary to conclude that the speed effect is peculiar to the induced motion situation and that, at higher speeds, configurational change becomes less effective because it is in conflict with ocular pursuit or with image displacement.

\section{REFERENCES}

BeCKLeN, R., W AlLACH, H. NitzberG, D. (1984). A limitation of position constancy. Journal of Experimental Psychology: Human Perception and Performance, 10, 713-723.

Coren, S., Bradley, D. R., Hoenig, P., \& Girgus, J. S. (1975). The effect of smooth tracking and saccadic eye movements on the perception of size: The shrinking circle illusion. Vision Research, 15, 49-55.

Wallach, H., BacoN, J., \& SChulman, P. (1978). Adaptation in motion perception: Alteration of induced motion. Perception \& Psychophysics, 24, 509-514.

WALLACH, H., BECKLEN, R. (1983). An effect of speed on induced motion. Perception \& Psychophysics, 34, 237-242.

Wallach, H., O'LEARY, A., \& MCMAHON, M. L. (1982). Three stimuli for visual motion compared. Perception \& Psychophysics, 32, 1-6.

\section{NOTES}

1. Individual ratios above 1 were corrected according to the formula 2 - real diameter/estimated diameter before means were calculated. See Wallach and Becklen (1983).

2. One may object to this description of the display. Since the dot pattern provides identical landmarks for the vertical motion of the dot and for its horizontal relative displacement that results from the horizontal motion of the pattern, the two objective motions should result in a single configurational change that is the resultant of the vertical and the horizontal relative displacements. This would be a valid objection to speaking of two configurational changes if the two motions were in phase: a single configurational change would take place that would result in an oblique motion of the dot. But, since the two motions were combined with a $90^{\circ}$ phase shift and were simple harmonic, one motion was always at its highest rate when the other was at its lowest rate. This, we believe, justifies considering the resultant circular or oval path as consisting of two components, each of which is given semi-independently by configurational changes that are oriented at right angles to each other.

3 . Why there is a speed effect when circular motion is given by ocular pursuit whereas there is none for straight motion remains an open question.

(Manuscript received April 2, 1984; revision accepted for publication February 4, 1985.) 